Article

\title{
Recognition of Tactile Attribute Strength and Category Using Convolutional Neural Network
}

\author{
Peng Zhang ${ }^{1, *}$, Guoqi Y $\mathbf{u}^{2}{ }^{2}$,Dongri Shan ${ }^{1}$,Zhenxue Chen ${ }^{3}$ and Xiaofang Wang ${ }^{1}$ \\ 1 School of Electronic and Information Engineering, Qilu University of Technology (Shandong Academy of \\ Sciences),Shandong Province,China; shandongri@qlu.edu.cn(D.S.); wxf2012@stu.xjtu.edu.cn (X.W.) \\ 2 School of Mechanical and Automotive Engineering, Qilu University of Technology (Shandong Academy of \\ Sciences),Shandong Province,China; 1043119037@stu.qlu.edu.cn \\ 3 School of Control Science and Engineering, Shandong University,Shandong Province,China; chenzhen- \\ xue@sdu.edu.cn \\ * Correspondence: zp@qlu.edu.cn
}

\begin{abstract}
Objectives: In order to solve the problem that most of the existing research focuses on the binary tactile attributes of objects, which ignores the tactile attribute strength and category recognition, an attribute strength and category recognition method based on convolutional neural network matrix-label is proposed. Methods:Firstly, in the data preparation stage,we preprocess the raw data and determine the matrix labels to build the haptic dataset.Secondly,in the feature extraction stage,we fuse the haptic data of two fingers and use the convolutional neural network to extract the attribute strength features.Finally,in the attribute strength and category recognition stage,all channel haptic data is fused to predict the attribute strength and category.Results:We compared with the multi-label convolutional neural network method in terms of elastic strength,hardness strength and category, and compared the attribute strength recognition capabilities of the two methods using novel objects outside the haptic dataset.The results show that the accuracy of the last 20 iterations of the matrix-label method has an average elastic strength of $96.73 \%$,hardness strength of $97.34 \%$, and category of $96.67 \%$.The performance is better. When the Euclidean distance between the prediction of the novel object and the real label is less than 1,the accuracy of the elastic strength is best to reach $100 \%$, and the hardness strength is best to reach $100 \%$. The performance is better. Conclusions:The effectiveness of the method has been verified.Comparing with the convolutional neural network method,our method can effectively recognize the attribute strength and category of objects.
\end{abstract}

Keywords: robot tactile; convolution neural network; attribute strength identification; category identification; robot operating system

\section{Introduction}

When modeling the diversity of objects based on deep learning methods, such as image classification of visual data [1] and speech recognition of audio data [2], it is often necessary to pay attention to the differences among objects. In the field of haptics, the difference of haptic signals often reflects the difference of the properties of different measured objects, which is an important basis for classifying the input into discrete categories.

Tactile sensors can provide the robot with information about the interaction forces and the object itself [3]. For example, Whether the sensor is in contact with the object [4], whether the object slides [5], the physical properties of the object such as temperature [6], roughness and texture $[7,8]$. The robot collects this information through a dynamic [9] exploration program (EPs) to get haptic data from the object. Collected perceivable tactile 
properties depend on EP [10]. In general, a lot of original data is needed in the deep learning model for training. these data needs to be artificial markers, which makes the model to a specific set.The model can only identify objects that have a certain amount of data in the set.For this problem, learning higher-level attributes of objects, such as elasticity, hardness and roughness of objects, may be more conducive to model generalization.

Many researchers have tried to teach robots to recognize tactile adjectives of objects from raw data and map the features of haptic data to artificially labeled data labels by using binary tactile adjectives (e.g., hard or not hard) to label objects [11, 12]. However, the above approach has the following disadvantages:

- In the existing open dataset $[11,12]$, the experimenter was only required to give the binary label (yes/no) of each object's attribute, and the binary label does not contain the delicate recognition of the object's attribute, so it is impossible to evaluate the strength of the object's attribute.

- In the real scene, human beings have a rich tactile perception system. Touching an object will get a delicate sensory feedback and have a specific cognition of the strength of the object's attributes. However, for robot tactile perception, only using binary tactile labels to describe objects will simplify the object attributes to binary space [13], which makes the robot have a very rough cognition of the strength of the object attributes.

This paper aims to enable the robot not only to recognize the tactile properties of objects, but also to understand the strength of the properties of objects more delicately, and then to recognize the categories of objects. Therefore, in order to solve the shortcomings of the above existing studies, this paper uses Kinova manipulator and NumaTac tactile sensor to establish the haptic dataset of the intensity of object attributes. The dataset contains two parts: the haptic sample of the object and the corresponding matrix label of the sample. Among them, the haptic sample contains force signal and vibration signal, and the matrix label contains the elastic strength and hardness strength information of the object attribute. Then, a convolutional neural network $(\mathrm{CNN})$ based object attribute strength and category recognition algorithm is proposed, which is used to identify the elastic strength, hardness strength and object category of the object in the haptic dataset. Through experiments, while ensuring a high recognition accuracy of the attribute intensity and category of objects in the dataset, some haptic samples of objects outside the dataset are selected to verify the adaptive attribute intensity recognition ability of this method, and a good effect is obtained.

The structure of this paper is as follows: In Section 2, the latest progress related to this topic is introduced. In Section 3, the process of establishing the haptic dataset is introduced. Section 4 introduces the algorithm of object attribute strength and category recognition based on convolutional neural network. Section 5 presents the experimental results of the recognition algorithm on the haptic dataset. In Section 6, we give the discussion about the results. Finally, in Section 7, we summarize the whole paper and the future work.

\section{Related Work}

The robot's tactile object recognition capability is achieved by processing haptic signals, which can be represented as a continuous signal, a set of discrete measurements, or a series of images [14].Different data structures may affect how the data is processed. From the perspective of the data structure of haptic signals, this paper reviews the related studies on the use of haptic data to identify objects and their attributes.

At present, the common haptic signals are force signals and vibration signals. If tactile sensors provide a global pressure value, then the best form of haptic signals may be a time-varying pressure curve signal, which can be used to detect contact or sliding events between contact surfaces $[15,16]$. If the sensor is made up of a set of electrodes or sensing units [17], then haptic images may be a good signal representation. Some studies only consider the static information of the pressure images [18]. Although the object is pressed 
several times [19], each pressure image only contains the pressure distribution information caused by the shape of the object, and does not contain the time relationship between the images. Therefore, some research will each image represents a transient moment pressure readings matrix, and haptic image sequence can contain the physical properties of a object information changes over time [20]. For haptic image, machine learning methods, such as k-nearest neighbor [21], bayesian method [22] and the traditional method based on image $[23,24]$ was used to identify features. But this does not mean that haptic data must use these corresponding methods.

There has been some progress in deep learning using haptic data to identify objects [25]. In [26], a recognition system using deep learning method and tactile sensor is proposed, and this system can recognize objects by grasping objects and cannot recognize the physical properties of objects, In [27], a multi-class and multi-label deep learning model is designed. The model can simultaneously learn four tactile features, including hardness, thermal conductivity, roughness and texture from haptic images, and identify targets according to these features. Considering the good performance of $\mathrm{CNN}$ processing to extract spatial features, the use of CNN to process tactile images is widely used [28, 29]. In addition, some researchers use advanced processing of haptic pressure images to classify and recognize objects. For example, the pressure images obtained during extrusion and release are connected into a tensor that can be used to classify objects in 3D CNN [30].

Regardless of the form of the data structure of the haptic signal, one way to get richer information about the haptic signal is for humans to classify haptic samples using discrete categories that are more detailed than binary decisions. The simplest partitioning task is to sort objects, classify them according to their similarity, and select one or more dimensions for analysis. In [31], The results of free sorting of different material samples were analyzed by multidimensional scaling, and the tactile material space was calibrated by physical measurements of compressibility and roughness. Similarly, [32] discusses the main dimensions of tactile surface perception. Roughness, smoothness and hardness are important orthogonal dimensions, and it is concluded that elasticity may correspond to the third main dimension. However, in a later study [33], the third major dimension of tactile perception was identified as viscosity/slippage. In addition, in [11, 12], a number of different researchers were selected to give binary ratings to adjectives of different objects, but the antisense relationships between adjectives were not taken into account. Therefore, [13] complements this aspect of the study by confirming the antonym pairs of hard/soft, rough/smooth and cold/warm, And more tactile information than binary label is analyzed.

\section{Materials}

The haptic datasets:Penn Haptic Adjective Corpus-1(PHAC-1) and Penn Haptic Adjective Corpus-2(PHAC-2) are proposed by using BioTac sensors in [11, 12]. However, each volunteer was only required to give the binary label (yes/no) of each object attribute, and the binary label cannot judge the strength of the object attribute. Therefore, this paper uses its own haptic data collection platform to establish the haptic dataset of the strength of the object attribute.

\subsection{Robot platform}

As shown in Figure 1, the desktop Kinova robotic arm equipped with Syntouch's NumaTac tactile sensor is selected as the haptic data acquisition platform in this paper. 


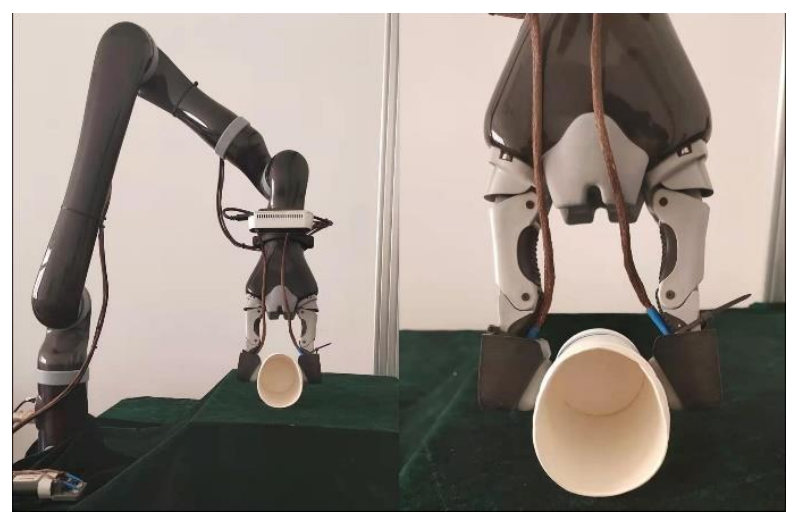

Figure 1. Robot operating platform that is picking up paper cup

The Kinova arm has seven degrees of freedom, including a 6-DOF manipulator and a 1-DOF two-finger gripper. Two NumaTac tactile sensors are mounted on each finger of the two-finger gripper. NumaTac tactile sensor is shown in Figure 2. The original data collected by it includes DC pressure signal $\left(P_{D C}\right)$ at a sampling frequency of $100 \mathrm{~Hz}$ and AC pressure vibration signal $\left(P_{A C}\right)$ at a sampling frequency of $2200 \mathrm{~Hz}$.

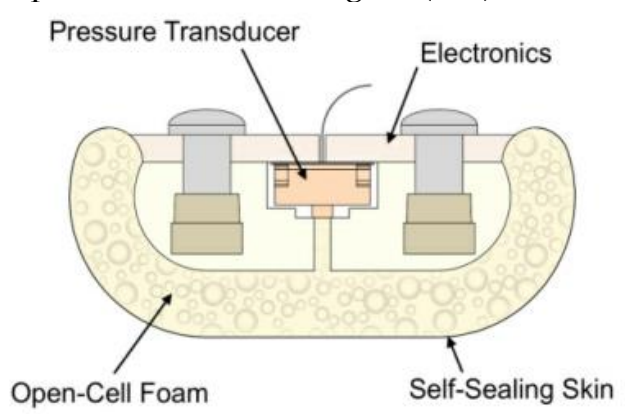

Figure 2. The NumaTac

The DC pressure value and AC pressure value per unit area can be calculated with Equations (1) and (2) :

$$
\begin{aligned}
& \mathrm{DC}=\left(P_{D C} \text {-offset }\right) \times 12.94 \mathrm{~Pa} / \mathrm{bit} \\
& \mathrm{AC}=\left(P_{A C} \text {-offset }\right) \times 0.13 \mathrm{~Pa} / \mathrm{bit}
\end{aligned}
$$

In Equation (1), offset is the DC pressure signal value of NumaTac tactile sensor signal under atmospheric pressure. In Equation (2), offset is the AC pressure vibration signal value of NumaTac tactile sensor signal under atmospheric pressure. $P_{D C}$ and $P_{A C}$ are instantaneous DC pressure signal values and instantaneous AC pressure vibration signal values obtained by NumaTac tactile sensor when the two-finger gripper interacts with the object, and the unit is bit.

The haptic data acquisition platform uses the Robot Operating System (ROS) as the software interface. ROS has a series of libraries and tools to help developers write Robot software programs, known as ROS nodes. When completing a complex task through a series of programs, ROS creates a network connecting all nodes, which is called ROS diagram. Nodes interact with each other to obtain information published by other nodes through the ROS diagram.

In this paper, the ROS diagram mainly included the Kinova Node, Syntouch Node and Data Processing Node, and the ROS Master managed the registration, communication and parameter servers of the node, as shown in Figure 3. 


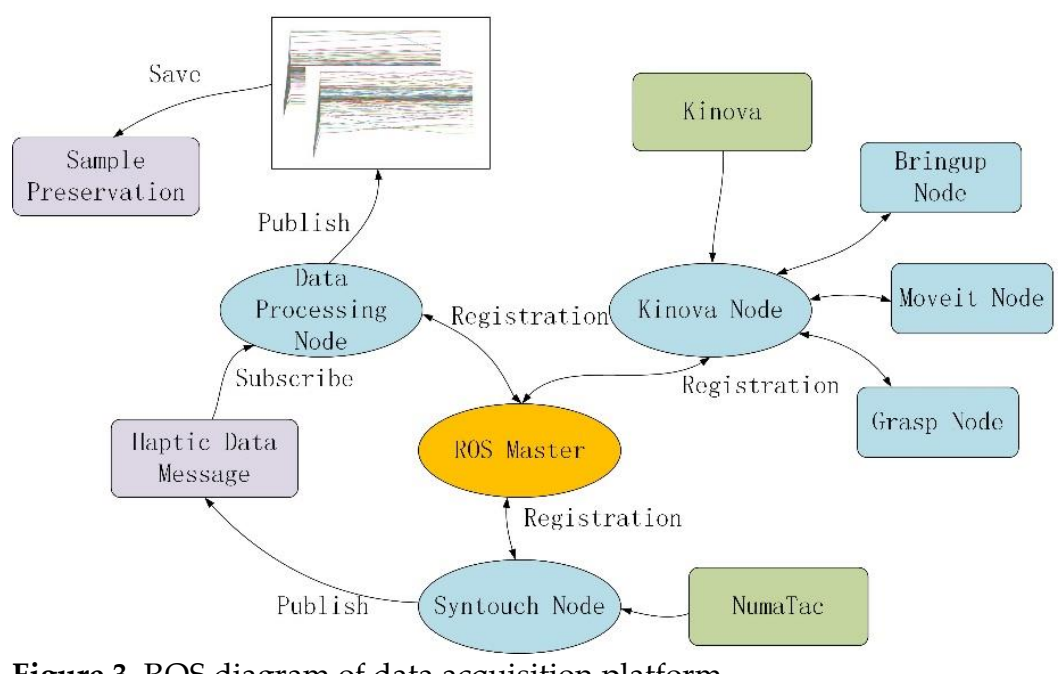

Figure 3. ROS diagram of data acquisition platform

- $\quad$ ROS Master is the core of ROS. It registered the names of nodes, services and topics and maintains a parameter server.

- Kinova Node is used to control the Kinova manipulator to move to the preset position and control the two-finger gripper to perform exploratory actions on the object. It consisted of three parts: the Bringup node to start all the nodes, to start some configurations for Kinova, and to activate the Kinova arm and the NumaTac tactile sensor; Moveit node starts the motion planning control component of the manipulator; Grasp node drives the manipulator arm to perform EPs on the target object.

- $\quad$ The Syntouch Node is used to publish the haptic data generated by the interaction between the NumaTac tactile sensor and the object. During the execution of a complete exploration action to generate haptic data, two NumaTac tactile sensors follow the two-finger gripper to physically interact with the object. The NumaTac tactile sensor data is continuously acquired and published to the ROS network at a frequency of $100 \mathrm{~Hz}$.

- The Data Processing Node subscribed to the haptic data released by the Syntouch node, and superimposed the data generated by two NumaTac tactile sensors released by the Syntouch node into a dual-channel haptic sample. Since Kinova manipulator first needs to reach the preset position before performing the exploration action, the NumaTac tactile sensor returns useless data. To solve this problem during this process, a dual thread is established. Firstly, the haptic data is continuously received in the main thread and it is judged whether the NumaTac tactile sensor has an initial contact with the object. When the initial contact is generated, the branch thread is started. The branch thread first waits for 3s until all the sample data in the main thread is received, and then intercepts the data and saves it as the haptic sample.

\subsection{NumaTac Haptic Dataset}

In this paper, the above haptic data acquisition platform is used to repeatedly interact with some common objects in daily life to collect a large number of haptic samples. After that, four experimenter is asked to interact with the objects physically to provide the elastic strength and hardness strength of each object to establish the object matrix label, and the haptic samples and matrix labels constitute the NumaTac Haptic Dataset (NHD).

\subsubsection{Objects}

Considering the foam material on the surface of the NumaTac tactile sensor and the motion limitations of the Kinova two-finger gripper. In this paper, as shown in Figure 4, 32 different objects are selected after excluding objects with dangerous characteristics such 
as sharpness, high temperature, humidity and unsuitable size. These 32 objects contain a variety of material properties and represent a wide range of physical properties.

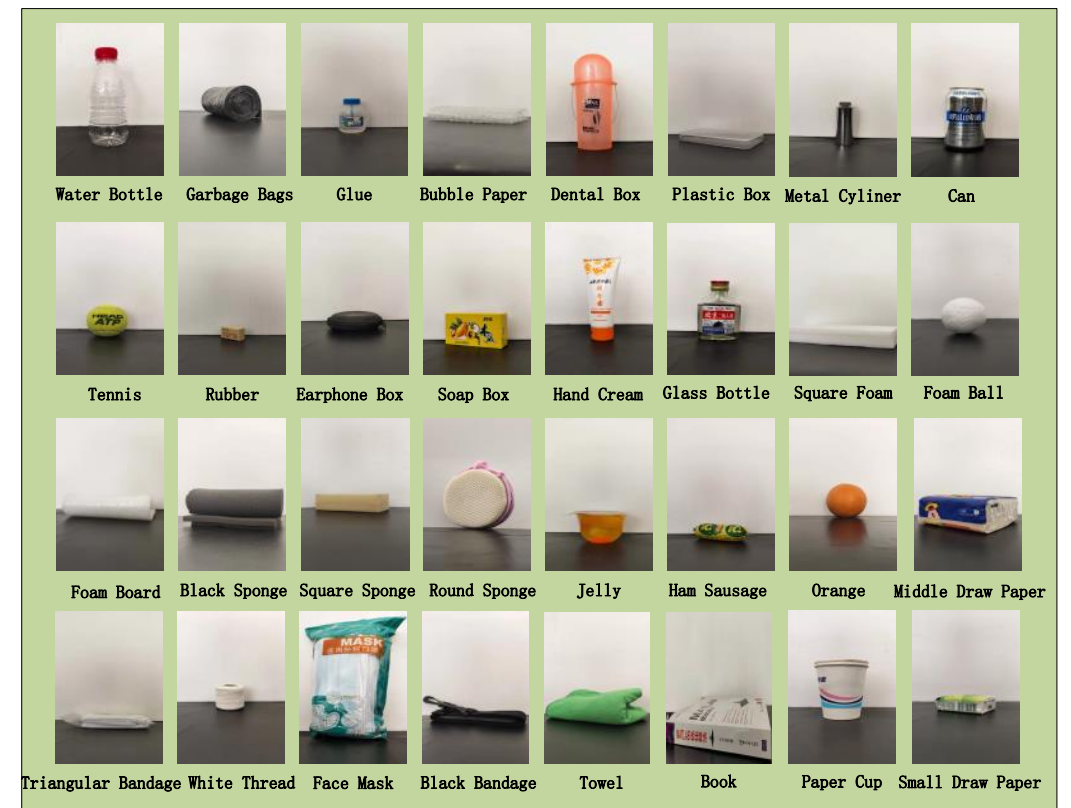

Figure 4.32 object pictures in NHD

\subsubsection{Data collection}

In general, humans use a series of exploratory actions when evaluating objects. Similarly, the movements of Kinova's robotic arm to explore the object in this paper are determined as: Squeeze, hold and release. Since the interaction between the robotic arm and the object are a continuous process. the above three exploratory actions are combined into a complete process to collect haptic data.

The Kinova arm performs these explorations on each object to obtain tactile information. Through adjusting the object location, the object are kept on the central axial plane of the two-finger gripper, setting up small posture change of uncertainty, and the squeezing action occured so that both fingers of the gripper contact the object at almost the same time, which prevent the object centroid position on two fingers of the tactile sensor interference and reduce unnecessary changes between experiment.

When the exploration begins, the grippers hand is closed at a uniform speed until the NumaTac tactile sensor on the two fingers make initial contact with the object. At the beginning of the object squeezing stage, NumaTac tactile sensors continue to deform to obtain haptic signals until a force threshold of $3493.8 \mathrm{~Pa}$ is reached, i.e. the $P_{D C}$ value of one of the NumaTac tactile sensors reaches 270bits (offset reaches 238), and the callback function in the node sends a stop command to the grippers. Because the signal takes time to transmit , the gripper are going to continue to squeeze the object and send back data for a short time before receiving the stop command, but this situation is more realistic. When the object squeezing stage is over, the gripper enter the object holding stage. The opening angle of the gripper remains the same, and the NumaTac tactile sensor deforms unchanged to receive the haptic signal for a period of time. At the end of the object holding stage, the gripper opens at a uniform speed and enters the object release stage. The NumaTac tactile sensor returns to its initial shape and state and generats haptic signals.

The haptic data acquisition platform is used to perform the above continuous exploration actions to touch 32 kinds of objects, each object is touched 50 times, and all $P_{D C}$ and $P_{A C}$ is recorded.

\subsubsection{Data preprocessing}


The approximate time required for a complete data collection operation is 35 , and the frequency of the NumaTac haptic data returned to the ROS network is $100 \mathrm{~Hz}$, so the length of each sample data is set to 300 discrete data points.

Considering the difference in data distribution between $P_{D C}$ and $P_{A C}$, the data are normalized according to Equation (3). Then, looking at the haptic data collected and taking into account the symmetry of the mechanical structure when the dexterous robotic hand grabs the object, In order to retain the integrity of the tactile information of the measured object and increase the characteristic quantity of the tactile information of the sample, the haptic data of the left and right fingers of each sample are superplaced into a double-channel sample.

$$
x^{*}=\frac{x-\mu}{\sigma}
$$

In Equation (3), $x^{*}$ is the normalized data, $\boldsymbol{x}$ is the original data, $\mu$ is the mean value of $\boldsymbol{x}, \sigma$ is the standard deviation of $\boldsymbol{x}$.

\subsubsection{Label}

In order to match the haptic data of the object with its real label, four subjects are found, and then put on an eye mask and a headset. In the blind environment, two fingers are used to simulate the exploratory movement of the robot dexterous hand touching the object, and the strength of the object attribute is compared in pairs one by one. Then, all the experimenters are asked to give the grade of elastic strength and hardness strength for each object, and the grade range is limited to 10 grades from 1 to 10 . After that, the grade is transformed into a binary label of $1 \times 10$ with a value of 1 at the strength level and 0 at the other positions. Finally, the point of value 1 is taken as the intersection point. The binary labels are vertically crossed into a $10 \times 10$ matrix, which contained the elastic strength label, hardness strength label and category label of the object. As shown in Figure 5 ,it is the location map of all objects, and the matrix element of the object's location is at the strength level value 1 . The $10 \times 1$ column vector of the location of the object is used as the elastic strength label, the $1 \times 10$ row vector is used as the hardness strength label, and the entire $10 \times 10$ matrix is used as the category label.

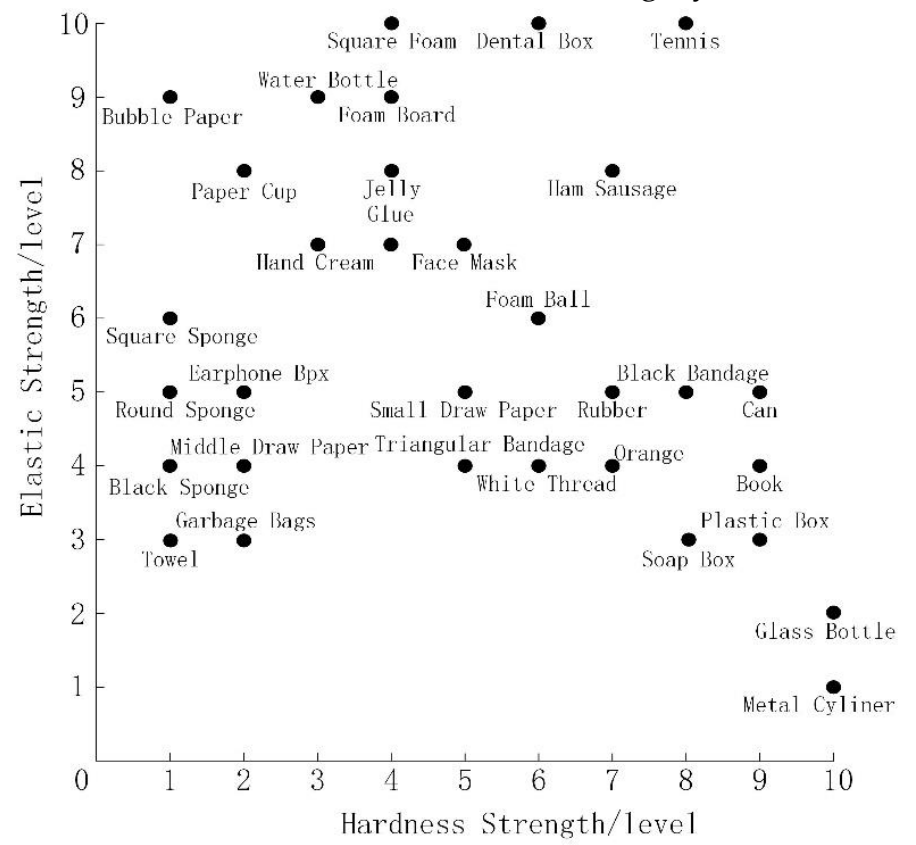

Figure 5. Location map of 32 objects in the matrix in NHD

For the problem of different intensity levels given by different experimenters, the experimenters can vote on all the intensity levels given, and the experimenters could agree with their own or others' judgment of the object. This process is completely decided by the internal discussion of the four experimenters, without the influence of other external 
personnel. The experimenters is required to give unique elastic strength and hardness strength rating for each object that will not be changed.

\section{Method}

It is an important problem that tactile attribute strength and category recognition of objects, One way to obtain rich tactile information similar to human perception is to use more discrete categories to represent the strength of an object's attributes. According to the similarity of attributes and the difference of attribute strength among different objects, the objects are sorted to determine the strength of each attribute of each object, and then the category of objects is determined by the strength of all the attributes of the object., After that, the category of the object is determined by the intensity of all the attributes of the object. This process of determining the intensity of the attributes and the category label of the object needs to be completed jointly by several experimenters in the real scene. The feature extraction of tactile information is the basis of the recognition of the strength and category of the object's attributes. Considering the good feature extraction ability of CNN, this paper uses CNN to extract the tactile information features of the object, and used matrix-label to represent the elastic strength, hardness strength and category of the object. The structure and parameters of matrix-label CNN are shown in Figure 6.

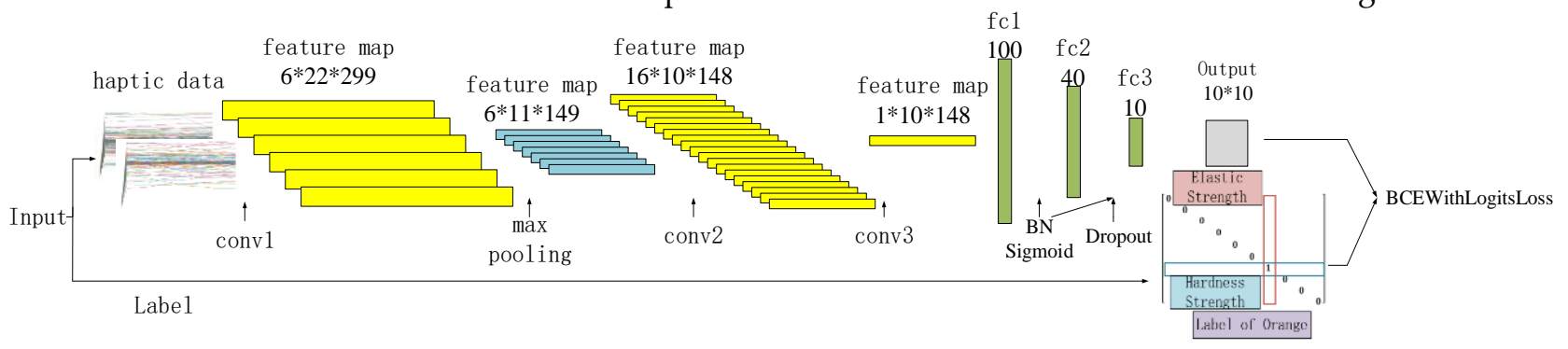

Figure 6. The structures of matrix-label CNN

CNN are born for images, but its application are not limited to images, so the characteristic information of haptic data are extracted through the above matrix-label CNN, the relationship between the data are analyzed, and the parameters are optimized in the training process, as follows:

- Input consists of two parts: one is a data layer that represented the object's haptic dataset. Each haptic sample is composed of data superimposed from two tactile fingers. The other part represents the real matrix-label of the object. Take an orange as an example, as shown in Figure 6 , the real label contained the orange's elastic strength (red box), hardness strength (blue box) and category label (matrix).

- The conv1, conv2 and conv3 are the feature extraction layer. In order to match the output of the network with the dimension and scale of the matrix-label, the last convolution layer is set with $1 \times 1$ convolution kernel and single output channel.

- The max pooling has the function of maximum pooling, which reduced the parameters of the neural network and improved the calculation speed.

- The fc1、fc2 and fc3 map the learned features to the sample mark space; Normalize the data in the BN layer; Sigmoid activation function is used to map the data within the range $[0,1]$. In order to prevent overfitting, Dropout regularization technology is adopted [34].

- Output a $10 \times 10$ matrix to calculate the distance from the real label.

- The loss function is BCEWithLogitsLoss. Equation (4) is the calculation formula of BCEWithLogitsLoss.

$$
\text { BCEWithLogitsLoss }=-\frac{1}{N} \sum_{i=0}^{N}\left(\boldsymbol{w}_{i}\left[\boldsymbol{y}_{i} * \log \sigma\left(\boldsymbol{x}_{i}\right)+\left(1-\boldsymbol{y}_{i}\right) * \log \left(1-\sigma\left(\boldsymbol{x}_{i}\right)\right)\right]\right)
$$

In Equation (4), $\boldsymbol{i}$ is the number of elements in the matrix label, $\mathrm{N}$ is the total number of elements in the matrix label, $\boldsymbol{w}_{\boldsymbol{i}}$ is the weight of the neural network, $\boldsymbol{y}_{\boldsymbol{i}}$ is the matrix 
label of the sample, $\boldsymbol{x}_{\boldsymbol{i}}$ is the predicted output of CNN network, $\sigma$ was the Sigmoid activation function.

Before starting the training, network parameters are initialized and parameters of the convolutional layer and the full connection layer are initialized to a normal distribution with an average value of 0 and a standard deviation of 1 .

\section{Experiment and Results}

\subsection{Experiment and Analysis of Object Attribute Strength and Category Recognition}

In this paper, PyTorch, a deep learning library based on Python, is used to build a neural network, and all haptic samples in NHD are trained and tested. Moreover, the matrix label CNN method is compared with the multi-label CNN method in terms of attribute intensity perception and object recognition effect.

Take an orange as an example, orange elastic strength, hardness strength and category label form of multi-label CNN method are shown in Figure 6.

\begin{tabular}{|c|c|c|c|c|c|c|c|c|c|c|c|c|c|c|c|c|c|c|c|}
\hline & & & las & & st & ens & & & & & & $\mathrm{Ha}$ & dn & SS & st1 & eng & & & \\
\hline$[0$ & 0 & 0 & 0 & 0 & 0 & 1 & 0 & 0 & 0 & 0 & 0 & 0 & 0 & 0 & 0 & 1 & 0 & 0 & $0]$ \\
\hline
\end{tabular}

Figure 7. Elastic strength, hardness strength and category label of orange

In the experimental hyperparameter setting, all haptic samples in NHD are randomly divided into training set and test set at a ratio of $4: 1$. The Adam optimizer is used to set the initial learning rate as 0.05 , the batch training quantity is 64 samples, and the total iteration training is 80 times. The recognition accuracy of training and testing of each method is shown in Table 1.

Table 1.Comparison of elastic strength, hardness strength and category recognition Accuracy rate of each method

\begin{tabular}{ccccc}
\hline Method & & Elastic Strength & Hardness Strength & Category \\
\hline Matrix-Label & Train & $99.20 \%$ & $99.17 \%$ & $99.16 \%$ \\
CNN & Test & $96.73 \%$ & $97.34 \%$ & $96.67 \%$ \\
& & & & \\
Multi-Label & Train & $99.74 \%$ & $99.79 \%$ & $99.60 \%$ \\
CNN & Test & $95.64 \%$ & $96.52 \%$ & $94.44 \%$ \\
\hline
\end{tabular}

It can be seen from Table 1 that, compared with the use of multi-label CNN, the accuracy of the test is relatively high in the identification of the elastic strength and hardness strength of the object, as well as in the result of category identification, which increases by $1.09 \%, 0.82 \%$ and $2.23 \%$ respectively. In addition, the accuracy gap of training and testing using matrix-label CNN is smaller than that of multi-label CNN.

\subsection{Experiment and Analysis of Novel Object Attribute Strength Perception}

In real life, it is not only impossible to collect, but also impossible to pre-train that the haptic data of all objects. Recognition of untrained novel object attributes by trained object attributes $[35,36]$ usually required attribute correlation between objects. Inspired by this idea, and by the ability of matrix-label CNN method proposed to identify object attribute strength, this paper tested the attribute strength of 5 novel objects.

Considering the selection conditions of objects in NHD, five novel objects are selected, which are Badminton, Warm Ears, Hard disk Pack, Inkpad Box and Nail Box. The test results of matrix-label $\mathrm{CNN}$ and multi-label $\mathrm{CNN}$ on the identification of the strength of the novel object's attribute and the occurrence times of the strength of the attribute are shown in Table 2 and Table 3 respectively.

Table 2. Recognition results of elastic strength and hardness strength of novel objects based on matrix-label CNN 


\begin{tabular}{cll}
\hline \multirow{2}{*}{ Object } & $\begin{array}{c}\text { Attribute } \\
\text { Strength }\end{array}$ & \multicolumn{1}{c}{ Pre-out/[strength (frequency) $]$} \\
\hline \multirow{2}{*}{ Badminton } & Elastic Strength & {$[10(2), 8,7,6(9), 5(26), 4(7), 3(3), 2]$} \\
& Hardness Strength & {$[1(3), 2(5), 3,7,8(37), 9(3)]$} \\
Warm Ears & Elastic Strength & {$[3(18), 2(2)]$} \\
& Hlardness Strength & {$[2(48), 4,9]$} \\
Hard disk & Elastic Strength & {$[8(4), 6(19), 5(15), 4(10), 3(2)]$} \\
Pack & Hardness Strength & {$[1(33), 2(4), 3,5(12)]$} \\
\multirow{2}{*}{ Inkpad Box } & Elastic Strength & {$[8,3(49)]$} \\
& Ilardness Strength & {$[7,8(49)]$} \\
Nail Box & Elastic Strength & {$[10(22), 9(4), 6(2), 5(13), 4(9)]$} \\
& Ilardness Strength & {$[1(38), 5(11), 6]$} \\
\hline
\end{tabular}

Table 3. Recognition results of elastic strength and hardness strength of novel objects based on multi-label CNN

\begin{tabular}{ccl}
\hline \multirow{2}{*}{ object } & \multicolumn{1}{c}{$\begin{array}{c}\text { Attribute } \\
\text { Strength }\end{array}$} & Pre-out/[strength (frequency) $]$ \\
\hline \multirow{2}{*}{ Badminton } & $\begin{array}{c}\text { Elastic Strength } \\
\text { Hardness Strength }\end{array}$ & {$[10(49), 5]$} \\
& Elastic Strength & {$[9,6,3(12), 8(38)]$} \\
Warm Ears & Hardness Strength & {$[1(9), 2(41)]$} \\
& Elastic Strength & {$[10(9), 9,7(19), 6(4), 5(11), 4(5), 3]$} \\
Hard disk & Hardness Strength & {$[1(19), 2(2), 5(22), 6(7)]$} \\
Pack & Elastic Strength & {$[9,8,7(5), 3(35), 1(8)]$} \\
Inkpad Box & Hardness Strength & {$[3,5,7(2), 8(39), 10(7)]$} \\
& Elastic Strength & {$[9,4(49)]$} \\
Nail Box & Ilardness Strength & {$[2,4(2), 5(26), 7(16), 9(5)]$} \\
\end{tabular}

As could be seen from Table 2 and Table 3, when each novel object is tested for 50 times, various methods has a variety of prediction results for the strength of the object's attribute, and the occurrence times is different. However, each object has the strength with the most occurrence times, so it could be considered that the neural network has a tendency for the strength of the attribute.

In order to more intuitively display the recognition results of each method on the attribute strength of the novel object, the recognition accuracy of each method is judged by the Euclidean distance between the predicted value and the label. The recognition accuracy results of each method is shown in Figure 8 and Figure 9.

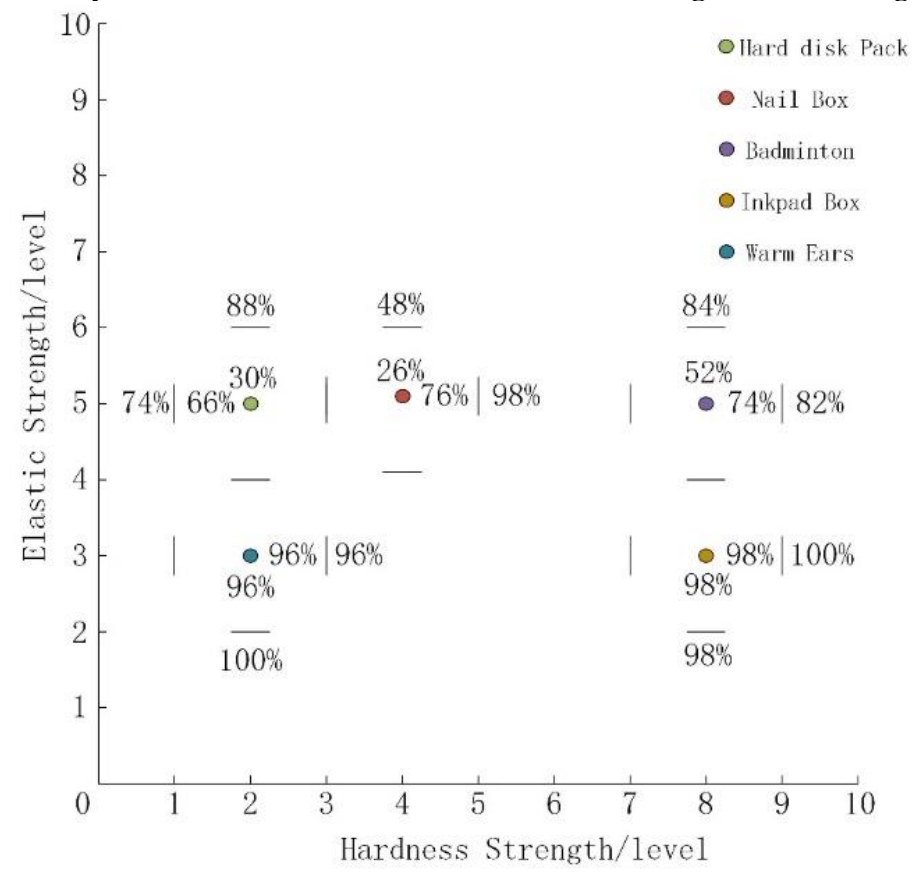

Figure 8. Matrix-label CNN accuracy chart based on euclidean distance 0 and 1 


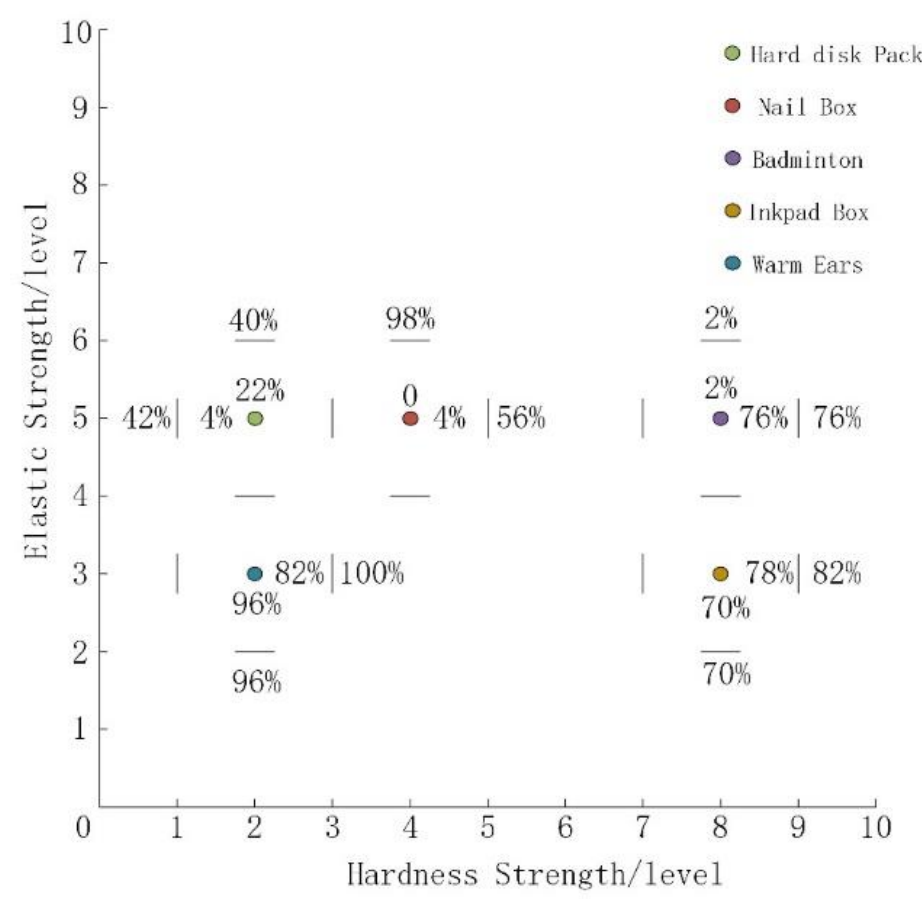

Figure 9. Multi-label CNN accuracy chart based on euclidean distance 0 and 1

Similar to the matrix-label determination process in the NHD, the same four experimenters are asked to judge the attribute strength of the five novel objects. In Figure 8 and Figure 9, the coordinates of the five dots are the elastic strength and hardness strength of the five objects jointly determined by the experimenters. The Euclidean distance between the attribute strength predicted by the neural network and the real attribute strength determined by the experimenter is used as the criterion of judgment, and two quantitative values of Euclidean distance 0 and 1 are selected. The $X$-axis value of the dot is the prediction accuracy of hardness strength under different Euclidean distances, and the $\mathrm{Y}$-axis value is the prediction accuracy of elastic strength under different Euclidean distances.

As can be seen from Figure 8 and Figure 9, except for Euclide distance 1, the accuracy rate of matrix-label CNN in testing Nail Box is $48 \%$ lower than that of multi-label CNN $(98 \%)$, and the accuracy rate of Warm Ears is $96 \%$ lower than that of multi-label CNN $(100 \%)$. All the others are matrix-label CNN with better testing effect on the strength of novel object attributes. In terms of elastic strength prediction, the best result is $100 \%$ accuracy of Warm Ears at Euclide distance 1, and the worst result is $26 \%$ accuracy of Nail Box prediction at Euclide distance 0, but the accuracy increased to $48 \%$ at Euclide distance 1. In terms of hardness strength prediction, the best result is $100 \%$ accuracy of Ink Box, and the worst result is the Hard disk Pack with $66 \%$ accuracy when the Euclide distance is 0 , but $74 \%$ when the Euclide distance is 1 .

From the above results, it could be seen that the matrix-label CNN method has a good ability to predict the attribute strength of novel objects, and its performance is better than that of the multi-label CNN method.

\section{Discussion}

There are abundant tactile sensors on human palms, so it is an interesting idea to carry tactile sensors on robotic arms. By designing manipulator arm control algorithm and object recognition algorithm, the intelligent perception and interaction level of the robot can be enhanced.

This paper aims to enable the robot not only to recognize the tactile properties of objects, but also to understand the strength of the properties of objects more delicately, and then to recognize the categories of objects. For this purpose, this paper uses Kinova manipulator and NumaTac tactile sensor to build the tactile attribute intensity dataset of 
the object, which includes the haptic data samples and matrix labels of the object. Among them, the signals in the haptic samples of objects are force signals and vibration signals that change at any time, and these two signals can well reflect the elasticity and hardness properties of objects. In addition, there must be a correlation between the properties of objects. As an important part of deep learning algorithms, labels in the haptic dataset exist in the form of matrix, which can better reflect the relationship between the data, which is obviously very interesting.

In this paper, a matrix-label CNN method is proposed to identify the attribute strength of an object. In order to verify the effectiveness of this method, this method is trained and tested by using the haptic dataset mentioned above. Experiments show that, compared with the multi-label CNN method, this method can better extract the haptic data features of the object, and can identify the elastic strength, hardness strength and object category of the object at the same time. In addition, the real world is a non-structural environment, so it is impossible to collect the haptic data of all objects. Therefore, some haptic samples of novel objects are tested. The results show that the matrix-label CNN has a good recognition effect on the strength of the object attribute, which proves the validity of the haptic dataset and the ability of the matrix-label CNN to recognize the strength of the object attribute. It is a good application direction to use robots to identify objects online, which may be carried out in future work.

\section{Conclusions}

In this paper, a 7-DOF robotic arm platform is used. The platform includes a 6-DOF robotic arm and a 1-DOF mechanical grip. Two fingers of the gripper are each equipped with a NumaTac tactile sensor. Through this operation platform, we set up an object attribute intensity haptic dataset, and select the object the elasticity and hardness of dimension analysis. Since our purpose is to make a delicate analysis of the properties of objects, a method to identify the elastic strength and hardness strength of objects is proposed based on $\mathrm{CNN}$, and the results prove that this method has a good effect.

In the future work, we suggest focusing on the relationship between tactile dimensions and a more delicate tactile attribute intensity classification method to identify the object intensity. In addition, how to apply it to the actual robot application is also a problem that should be considered.

Author Contributions: Conceptualization, G.Y.; methodology, G.Y.; software, G.Y.; validation, G.Y.; formal analysis, G.Y., X.W.; investigation, G.Y.; resources, P.Z.,D.S.; data curation, G.Y.; writingoriginal draft preparation, G.Y.; writing - review and editing, P.Z.,D.S.,Z.C. and X.W.; visualization, G.Y.; supervision, P.Z. and D.S.

Funding: This research was funded by Shandong Provincial Major Scientific and Technological Innovation, Grant No.2019JZZY010444 and No.2019TSLH0315, and National Key Research and Development Project, Grant No. 2019YFB1311001, and the Project of 20 Policies of Facilitate Scientific Research in Jinan Colleges, Grant No.2019GXRC063.

Data Availability Statement: https:/github.com/yuguoqi-learner/NHD-and-method-of-paper-.git

Conflicts of Interest: The authors declare no conflict of interest..

\section{References}

1. Deng, J.; Dong, W.; Socher, R.; Li, L. J.; Li, K.; Li, F. F.; Ieee, ImageNet: A Large-Scale Hierarchical Image Database. In Cvpr: 2009 Ieee Conference on Computer Vision and Pattern Recognition, Vols 1-4, 2009; pp 248-255.

2. Bai, Z. X.; Zhang, X. L., Speaker recognition based on deep learning: An overview. Neural Networks 2021, 140, 65-99.

3. Lederman, S. J.; Klatzky, R. L., Extracting object properties through haptic exploration. Acta Psychologica 1993, 84, (1), 29-40.

4. Komeno, N.; Matsubara, T., Tactile Perception Based on Injected Vibration in Soft Sensor. Ieee Robotics and Automation Letters 2021, 6, (3), 5365-5372. 
5. Zhang, Y.; Kan, Z.; Tse, Y. A.; Yang, Y.; Wang, M. Y., Fingervision tactile sensor design and slip detection using convolutional lstm network. arXiv preprint arXiv:1810.02653 2018.

6. Siegel, D.; Garabieta, I.; Hollerbach, J. M. In An integrated tactile and thermal sensor, IEEE International Conference on Robotics \& Automation, 1986; 1986.

7. Kaboli, M.; Cheng, G., Robust Tactile Descriptors for Discriminating Objects From Textural Properties via Artificial Robotic Skin. Ieee Transactions on Robotics 2018, 34, (4), 985-1003.

8. Tsuji, S.; Kohama, T., Using a convolutional neural network to construct a pen-type tactile sensor system for roughness recognition. Sensors and Actuators a-Physical 2019, 291, 7-12.

9. Lepora, N. F., Biomimetic Active Touch with Fingertips and Whiskers. Ieee Transactions on Haptics 2016, 9, (2), 170-183.

10. Okamura, A. M.; Cutkosky, M. R., Feature detection for haptic exploration with robotic fingers. International Journal of Robotics Research 2001, 20, (12), 925-938.

11. Chu, V.; McMahon, I.; Riano, L.; McDonald, C. G.; He, Q.; Martinez Perez-Tejada, J.; Arrigo, M.; Darrell, T.; Kuchenbecker, K. J., Robotic learning of haptic adjectives through physical interaction. Robotics and Autonomous Systems 2015, 63, (P3), 279292.

12. Chu, V.; McMahon, I.; Riano, L.; McDonald, C. G.; He, Q.; Perez-Tejada, J. M.; Arrigo, M.; Fitter, N.; Nappo, J. C.; Darrell, T.; Kuchenbecker, K. J. In Using robotic exploratory procedures to learn the meaning of haptic adjectives, Proceedings - IEEE International Conference on Robotics and Automation, 2013; 2013; pp 3048-3055.

13. Richardson, B. A.; Kuchenbecker, K. J., Learning to predict perceptual distributions of haptic adjectives. Frontiers in Neurorobotics 2020, 13.

14. Gandarias, J. M.; Gómez-de-Gabriel, J. M.; García-Cerezo, A. J., Enhancing perception with tactile object recognition in adaptive grippers for human-robot interaction. Sensors (Switzerland) 2018, 18, (3).

15. Su, Z.; Hausman, K.; Chebotar, Y.; Molchanov, A.; Loeb, G. E.; Sukhatme, G. S.; Schaal, S.; Ieee, Force Estimation and Slip Detection/Classification for Grip Control using a Biomimetic Tactile Sensor. In 2015 Ieee-Ras 15th International Conference on Humanoid Robots, 2015; pp 297-303.

16. Melchiorri, C., Slip detection and control using tactile and force sensors. IEEE/ASME Transactions on Mechatronics 2000, 5, (3), 235-243.

17. Briot, M., Utilization of an 'artificial skin' sensor for the identification of solid objects. Water Resources and Environmental Engineering Research Report (State University of New York at Buffalo, Department of Civil Engineering) 1979, 529-547.

18. Luo, S.; Mou, W. X.; Althoefer, K.; Liu, H. B., Novel Tactile-SIFT Descriptor for Object Shape Recognition. Ieee Sensors Journal 2015, 15, (9), 5001-5009.

19. Gandarias, J. M.; Gomez-de-Gabriel, J. M.; Garcia-Cerezo, A.; Ieee, Human and Object Recognition with a High-Resolution Tactile Sensor. In 2017 Ieee Sensors, 2017; pp 981-983.

20. Zapata-Impata, B. S.; Gil, P.; Torres, F., Learning Spatio Temporal Tactile Features with a ConvLSTM for the Direction Of Slip Detection. Sensors 2019, 19, (3).

21. Feng, J. H.; Jiang, Q., Slip and roughness detection of robotic fingertip based on FBG. Sensors and Actuators a-Physical 2019, 287, 143-149.

Pastor, F.; Garcia-Gonzalez, J.; Gandarias, J. M.; Medina, D.; Closas, P.; Garcia-Cerezo, A. J.; Gomez-de-Gabriel, J. M., Bayesian and Neural Inference on LSTM-Based Object Recognition From Tactile and Kinesthetic Information. Ieee Robotics and Automation Letters 2021, 6, (1), 231-238.

23. Okamura, A. M.; Turner, M. L.; Cutkosky, M. R. In Haptic exploration of objects with rolling and sliding, Proceedings - IEEE International Conference on Robotics and Automation, 1997; 1997; pp 2485-2490. 
24. Dong, S. Y.; Yuan, W. Z.; Adelson, E. H., Improved GelSight Tactile Sensor for Measuring Geometry and Slip. In 2017 Ieee/Rsj International Conference on Intelligent Robots and Systems, Bicchi, A.; Okamura, A., Eds. 2017; pp 137-144.

25. Schmitz, A.; Bansho, Y.; Noda, K.; Iwata, H.; Ogata, T.; Sugano, S.; Ieee, Tactile Object Recognition using Deep Learning and Dropout. In 2014 14th IEEE-RAS International Conference on Humanoid Robots, 2014; pp 1044-1050.

26. Yuan, W.; Zhu, C.; Owens, A.; Srinivasan, M. A.; Adelson, E. H. In Shape-independent hardness estimation using deep learning and a GelSight tactile sensor, Proceedings - IEEE International Conference on Robotics and Automation, 2017; 2017; pp 951958.

27. Han, D.; Nie, H.; Chen, J.; Chen, M.; Deng, Z.; Zhang, J., Multi-modal haptic image recognition based on deep learning. Sensor Review 2018, 38, (4), 486-493.

28. Lu; Jiwen; Abdulnabi; rar, A.; H.; Wang; Gang; Jia; Kui, Multi-Task CNN Model for Attribute Prediction. IEEE Transactions on Multimedia 2015.

29. Hui, W.; Li, H.; Chen, M.; Song, A., Robotic tactile recognition and adaptive grasping control based on CNN-LSTM. Yi Qi Yi Biao Xue Bao/Chinese Journal of Scientific Instrument 2019, 40, (1), 211-218.

30. Pastor, F.; Gandarias, J. M.; García-Cerezo, A. J.; Gómez-De-gabriel, J. M., Using 3d convolutional neural networks for tactile object recognition with robotic palpation. Sensors (Switzerland) 2019, 19, (24).

31. Bergmann Tiest, W. M.; Kappers, A. M. L., Analysis of haptic perception of materials by multidimensional scaling and physical measurements of roughness and compressibility. Acta Psychologica 2006, 121, (1), 1-20.

32. Holliins, M.; Faldowski, R.; Rao, S.; Young, F., Perceptual dimensions of tactile surface texture: A multidimensional scaling analysis. Perception Eamp; Psychophysics 1993, 54, (6), 697-705.

33. Hollins, M.; Bensmaïa, S.; Karlof, K.; Young, F., Individual differences in perceptual space for tactile textures: Evidence from multidimensional scaling. Perception and Psychophysics 2000, 62, (8), 1534-1544.

34. Srivastava, N.; Hinton, G.; Krizhevsky, A.; Sutskever, I.; Salakhutdinov, R., Dropout: A Simple Way to Prevent Neural Networks from Overfitting. Journal of Machine Learning Research 2014, 15, 1929-1958.

35. Lampert, C. H.; Nickisch, H.; Harmeling, S.; Ieee, Learning To Detect Unseen Object Classes by Between-Class Attribute Transfer. In Copr: 2009 Ieee Conference on Computer Vision and Pattern Recognition, Vols 1-4, 2009; pp 951-958.

36. Wang, X. L.; Ye, Y. F.; Gupta, A.; Ieee, Zero-shot Recognition via Semantic Embeddings and Knowledge Graphs. In 2018 Ieee/Cvf Conference on Computer Vision and Pattern Recognition, 2018; pp 6857-6866. 